\title{
M-Budget: la médecine ne doit pas devenir un bien de consommation
}

\author{
Remo Osterwalder \\ Dr méd., vice-président de la FMH, responsable du département Prestations et développement professionnel
}

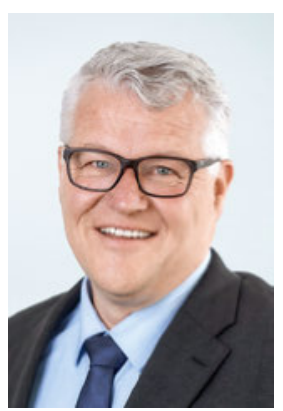

Le paysage des fournisseurs de prestations médicales se transforme à l'instar de la médecine elle-même en constante mutation. Autrefois, le modèle du médecin de famille et du cabinet particulier était légion, or nous constatons qu'entre-temps ce modèle n'est plus assez attrayant pour l'exercice de la profession. En guise d'alternative, les cabinets de groupe sont apparus sans être, eux non plus, en mesure de couvrir jusqu'ici tous les besoins en médecine de premier recours. Une autre forme de cabinet se développe désormais: les centres de santé avec des prestations interdisciplinaires. Mais pour différentes raisons, ils risquent d'occasionner des coûts moyens par patient qui pourraient être plus élevés que ceux d'un cabinet de groupe de médecins de famille. Des indicateurs qualité ont, jusqu'ici, fait défaut

\section{Le succès d'un modèle dépend de la bonne volonté des patients.}

pour voir par exemple si les taux d'hospitalisations sont identiques dans ces collectifs de comparaison ou si seules les prestations de base, et donc les coûts directs, sont comparés.

Une vague d'ouvertures de cabinets se propage désormais parmi les intéressés avec des motivations différentes. Les hôpitaux veulent s'assurer leur patientèle par l'acquisition de cabinets dans leur périmètre d'intervention, qu'ils confient ensuite à leur personnel. Si les effets indirects des hospitalisations ou les investigations par les cliniques spécialisées rattachées à l'hôpital concerné ne sont pas pris en compte, le taux d'efficience ne devrait pas être atteint. En effet, cette méthode ne permettant pas toujours de couvrir les coûts d'un cabinet particulier. Mais les hôpitaux ne sont pas les seuls, d'autres intéressés comme les assureurs investissent dans le marché de la santé. La volonté de maîtriser les coûts ne se limite pas aux seuls médecins. Il semble cependant que les assureurs s'étaient représentés différemment l'aventure avec les cabinets médicaux, sinon comment expliquer leur changement de stratégie concrétisé par la vente de leurs cabinets.
Aujourd'hui, un tout autre type de protagoniste se manifeste sur ce marché: la Migros. Au travers de ses centres de fitness, le géant orange est déjà bien implanté. Mais avec lui, le marché de la santé pourrait inaugurer une nouvelle ère, comme autrefois, lorsque Gottlieb Duttweiler a révolutionné le commerce de détail après la faillite de sa première entreprise en 1920 (Pfister \& Duttweiler) et l'achat des camions-magasins de la Migros, sa nouvelle société.

La relation de confiance entre le patient et son médecin constitue un préalable essentiel à tout traitement médical. Ici par exemple, le système de points Cumulus n'a pas sa place s'il s'agit de peaufiner la stratégie marketing sous couvert de médecine personnalisée. Autrement dit: la collecte des données personnelles de santé ne peut être utilisée qu'à des fins de promotion directe de la santé du patient et en dehors de tout intérêt commercial.

Attardons-nous brièvement sur la réalité du marché au quotidien: la majeure partie des coûts de la santé est occasionnée par une minorité de patients pendant une période relativement courte de la vie. Ce sont précisément eux qui posent un immense défi à la prise en charge médicale de demain. Les patients atteints de maladies chroniques souhaitent une seule personne de référence, sans que ce soit obligatoirement un

\section{Le système de points Cumulus n'a pas sa place} dans la relation médecin-patient.

médecin. Ainsi s'ouvrent d'énormes perspectives en matière d'interprofessionnalité. Pour le patient, il importe que cette personne de référence ne change pas en permanence et que la stratégie de traitement suivie soit cohérente. Or le succès d'un modèle dépend forcément de la confiance du patient et de la motivation de tous ceux impliqués dans le traitement. Laissons-nous surprendre et voyons si le modèle à succès de Gottlieb Duttweiler révolutionnera aussi le marché de la santé en Suisse. 